\title{
Surgical treatment of advanced endometrial carcinoma: multivisceral resection - literature review
}

\author{
Nicolae Bacalbașa ${ }^{1}$, A. Gavril ${ }^{2}$ \\ "Carol Davila" University of Medicine and Pharmacy, Bucharest, Romania \\ Emergency University Hospital „Elias” Bucharest, Romania
}

Corresponding author:

Nicolae Bacalbasa,

Dimitrie Racoviță Street, no. 2,

Bucharest, Romania

E-mail: nicolae_bacalbasa@yahoo.ro

\section{ABSTRACT}

Introduction: Complete resection is the most important prognostic factor in surgery for pelvic tumors. In locally advanced and recurrent pelvic malignancies, radical margins are sometimes difficult to obtain because of close relation to or growth in adjacent organs/structures. Total pelvic exenteration (TPE) is an exenterative operation for these advanced tumors and involves en bloc resection of the rectum, bladder, and internal genital organs (prostate/seminal vesicles or uterus, ovaries and/or vagina).

Methods: Recommendations with respect to extended surgical staging, provides important prognostic information and aids in determining the need for adjuvant treatments. Published literature was retrieved through searches of PubMed, CINAHL, and The Cochrane Library, using appropriate controlled vocabulary (e.g., endometrial neoplasms) and key words (e.g., endometrium cancer, endometrial carcinoma).

Results were restricted to systematic reviews, randomized control trials/ controlled clinical trials, and observational studies. There were no date or language restrictions. This review analyses the benefit of extended surgical staging compared with the potential harm of a limited surgery in grade 2 and 3 disease.

Conclusions: Total pelvic exenteration is accompanied with considerable morbidity, but good local control and acceptable overall survival justifies the use of this extensive surgical technique in most patients, especially patients with primary locally advanced or recurrent endometrial cancer.

Key words: endometrial cancer, lymphadenectomy, surgical staging, pelvic exenteration for endometrial cancer

\section{INTRODUCTION}

Locally advanced pelvic tumors without distant metastases can cause severe local problems, such as pain, voiding, and defecation problems, and may result in a decreased quality of life $(1,2)$. Extensive surgery often is the only possibility 
for complete resection, thereby attempting to provide local control and palliation. In case of local invasion of the urinary bladder trigone and of the rectal wall a total pelvic exenteration (TPE) providing en bloc resection of the tumor, rectum, urinary bladder, lower ureters, and internal genital organs could potentially salvage the patient (3). After resection of the urinary bladder, an ileal conduit is usually constructed according to the technique described by Bricker (4). TPE has been performed in primary or recurrent cancer of the cervix, rectum, vagina, uterine corpus, vulva, urinary bladder, or for pelvic sarcoma $(5,6)$.

In an era of organ preservation, exenteration is still an important armamentarium in selected cases of advanced pelvic malignancies. This is possible because certain cancers arising from pelvic viscera have the propensity for remaining confined to pelvic organs for a long time before metastasizing beyond pelvis (1). When first described by Brunschwig in 1948, the peri-operative mortality rate was $23 \%$ (2). Improvements in preoperative evaluation, antibiotic usage, anesthesia, surgical expertise and post operative care have all contributed to improved outcome and steady reduction in both morbidity and mortality. The ultimate goal is to bestow the patient a reasonable quality of life in addition to providing the chance for a cure (3).

\section{MATERIALS AND METHODS}

Surgery is a fundamental part of the management of endometrial cancer. The two principal goals of the surgery are (1) removal of the cancer, and (2) accurate documentation of the extent of disease (staging). While the former may be limited to simple hysterectomy and removal of the ovaries, the latter is much more involved and includes pelvic washings, omental biopsy or complete omentectomy, pelvic lymph node dissection, and paraaortic lymph node dissection.

In 1988, the International Federation of Gynecology and Obstetrics recommended that endometrial cancer be staged surgically. Controversy arose over what precisely constitutes an adequate staging procedure in endometrial cancer. A wide range of surgical procedures have been offered to patients, from simple hysterectomy and bilateral salpingo-oophorectomy to HBSO with omentectomy, and retroperitoneal nodal evaluation that includes both pelvic and para-aortic lymph node dissection.2 The 2009 revision of the FIGO staging recommendations further separates patients with pelvic nodal involvement (IIIC1) and para-aortic nodal metastasis (IIIC2), emphasizing the differences in prognosis between these two groups.

Currently, there are 2 commonly recommended options for surgical management of endometrial cancer patients: (1) limited surgery including a total hysterectomy and bilateral salpingo-oophorectomy, and (2) comprehensive surgical staging consisting of HBSO (hysterectomy and bilateral salpingo-oophorectomy) and surgical pathologic evaluation of retroperitoneal lymph nodes including pelvic and/or para-aortic regions, together with omentectomy in certain types of endometrial cancer. Individual centres and local disease site groups should determine treatment policies that are based on their own experience with accuracy of preoperative grading, availability and accessibility of preoperative MRI to determine depth of myometrial invasion, and ability to carry out intraoperative assessment of depth of myometrial invasion.

Steps to follow regarding the stage of the disease and the histological grade:

\section{Limited surgery (HBSO) in endometrial cancer}

Two recent prospective randomized trials have also demonstrated that lymphadenectomy offers no survival benefit in the management of endometrial cancer $(7,8)$. The ASTEC trial randomized 1408 patients with endometrial cancer (all grades included) to either HBSO with washings and removal of grossly enlarged lymph nodes or HBSO and complete surgical staging that included pelvic, but not para-aortic, lymphadenectomy (7). Adherence to the study protocol was good, but $45 \%$ of patients randomized to the lymphadenectomy arm had lymph node counts of less than 10 . The 5-year overall survival was $80 \%$ in the group that had full staging, and $81 \%$ in the group that had HBSO. Results were further confounded in the second part of the study, which randomized patients to receive pelvic radiation therapy regardless of their surgical-pathological findings. In 2008, Panici et al. looked at the value of lymphadenectomy in patient survival by conducting a prospective randomized trial of HBSO versus $\mathrm{HBSO}$ and lymph node dissection in patients with endometrial cancer (8).The protocol required that at least 20 pelvic lymph nodes be removed to be considered adequate evaluation in the lymphadenectomy arm. At a median follow-up of 48 months, the hazard ratio for death (lymphadenectomy vs. no lymphadenectomy arm) was $1.1(95 \% \mathrm{Cl} 0.7$ to $1.71 ; \mathrm{P}=0.50)$. The 5 -year diseasefree and overall survival rates were $81 \%$ versus $85.9 \%$ and $81.7 \%$ versus $90 \%$ with no significant differences between the two arms. 


\section{Surgical staging in endometrial cancer}

\section{Preoperative risk assessments for nodal involvement in edometrial cancer}

\section{Extent of lymph node evaluation in endometrial cancer}

\section{Lymphadenectomy based on clinical assessments}

\section{Lymph node count in endometrial cancer}

The largest study looking at lymph node counts was a review of 11443 endometrial cancer cases recorded in the SEER database between 1990 and 2001.44 Of the 11443 patients, 638 (5.6\%) had positive lymph nodes. Lymph node counts were analyzed categorically as follows: up to 5 nodes, up to 10 , up to 20 , up to 25 , and more than 25 nodes. The percentage of patients with positive nodes identified per category was $27 \%, 46 \%$, $74 \%, 85 \%$, and $100 \%$ respectively. A logistic regression model with a baseline category of 1 to 5 lymph nodes determined that the retrieval of 21 to 25 lymph nodes provided the greatest incremental gain in identification of positive lymph nodes (OR 1.45; $95 \% \mathrm{Cl} 1.08$ to 1.94; $\mathrm{P}<0.01$ ). Removing more than 25 lymph nodes did not significantly increase the probability of detecting at least 1 positive node. A 2008 Italian trial randomized patients with intermediate- and high-risk endometrial cancer to either HBSO or HBSO with lymph node dissection (8). The primary outcome was a comparison of overall survival between the 2 groups. The protocol required that at least 20 lymph nodes be removed for a lymphadenectomy to be considered adequate. Lymphadenectomies were carried out in the control arm of the study only if grossly enlarged lymph nodes were identified by palpation. Although there was no survival benefit in the lymphadenectomy arm of the study, statistically, more patients in this arm were found to have stage IIIC disease (13.3\% vs. $3.2 \%$ in the HBSO group; $95 \% \mathrm{Cl}$ 5.3\% to $14.9 \%$; $\mathrm{P}<0.001$ ).

\section{Sentinel lymph nodes in endometrial cancer}

\section{Advanced stage endometrial cancer}

The traditional approach to patients with advanced disease has been to offer palliative treatments (9). However, more recent approaches of surgically debulking patients with advanced endometrial cancer have shown improved survival when patients are optimally debulked $(9,10)$. In 2006, Randall et al. (11) demonstrated improved survival in patients with advanced disease who were optimally debulked to less than $2 \mathrm{~cm}$ residual disease when they subsequently received aggressive chemotherapy.

Consideration should be given to performing pelvic and/or para-aortic lymphadenectomies in patients with preoperative grade 2 and 3 disease to facilitate accurate planning of adjuvant therapy, which is often required postoperatively. The survival benefit of lymphadenectomy in this specific group of patients has not been studied. (II-2B) Patients with high-risk histological subtypes of endometrial cancer such as clear cell and papillary serous adenocarcinomas should receive full staging surgery that includes pelvic and/or para-aortic lymphadenectomy and omentectomy. (II-2B) A coordinated multidisciplinary team approach should be used for management of high-risk endometrial cancer. (III-B) Regional multidisciplinary teams should come to local consensus on the use of prognostic factors to guide adjuvant therapy. (III-B) Performing lymphadenectomy on the basis of palpation for "bulky" nodes is inaccurate and should not be done. Patients presenting with advanced disease should be referred to gynaecologic oncologists at a regional cancer centre for treatment planning. (II-2B)

Complete resection is the most important prognostic factor in surgery for pelvic tumors. In locally advanced and recurrent pelvic malignancies, radical margins are sometimes difficult to obtain because of close relation to or growth in adjacent organs/structures. Total pelvic exenteration (TPE) is an exenterative operation for these advanced tumors and involves en bloc resection of the rectum, bladder, and internal genital organs (prostate/ seminal vesicles or uterus, ovaries and/or vagina).

\section{DISCUSSION}

Brunschwig initially thought pelvic exenteration as a palliative procedure for locally advanced pelvic malignancies but the focus of the operation shifted towards cure (2). More than $50 \% 5$ year survival was noted following exenteration in many recent series $(3,9,12)$. Goldberg et al. (13) in their study concluded that indications for palliative exenteration should be exceptional and individualized, and that, in general, the procedure should not be undertaken. Nguyen et al. (14) demonstrated no survival benefit after pelvic exenteration done as a palliative procedure.

The complications of pelvic exenteration can be troublesome due to the residual defect resulting in a 
high incidence of postoperative small intestinal obstruction and fistula formation. Goldberg et al. (13) experienced a high rate of enteric (11/103) and ureteral anastomotic fistulas (14/103) while using mesh or other foreign material for pelvic floor reconstruction but no such complication with the rectus myocutaneous graft. In a study by Goldberg et al. (13) obliteration of the denuded pelvic cavity did not result in reduction of complications to a statistically significant level

Adjacent organ invasion significantly affected survival. Persistent or recurrent disease after RT, type of exenteration, nodal involvement within pelvis did not influence the survival to a statistically significant level. In a study by Berek et al. (3) no statistically significant correlation was found between the type of exenteration and overall survival. Lymph node involvement significantly affected survival after pelvic exenteration in some studies $(9,13)$.

The technique of total pelvic exenteration was introduced as a palliative procedure for patients with advanced gynecological cancer. Currently it is performed with curative intent in the treatment of locally advanced and recurrent pelvic disease (rectal, cervical, endometrial, vaginal, vulvar tumors, and soft-tissue sarcoma) (8). In locally advanced tumors (without distant metastasis), complete resection is the only possibility for cure

Barakat et al. described that surgery for recurrent endometrial cancer is associated with only $20 \%$ longterm survival and high morbidity (15). Preoperatively irradiated patients with locally advanced vaginal cancer are appropriate candidates for TPE with $>50 \%$ survival after 5 years according to Berek et al (3).

For many years, age was considered a contraindication for the performance of TPE. In the present study, patients older than aged 70 years had a similar outcome after TPE compared with younger patients, which is confirmed by other recent studies $(16,17)$. Not age but physical condition and comorbidities are considered important criteria in selecting patients for TPE.

\section{CONCLUSION}

Total pelvic exenteration is accompanied with considerable morbidity; however, good local control and acceptable overall survival justifies the use of this extensive surgical technique in selected patients with primary locally advanced and recurrent pelvic tumors. This analysis shows that role of exenteration is invaluable and can be done safely with acceptable complication rates and is the only curative hope in selected patients.

\section{REFERENCES}

1. Spratt JS1, Meyer JS. Biological considerations with pelvic neoplasms. J Surg Oncol. 1999 Jul;71(3):198-205.

2. Brunschwig A. Complete excision of pelvic viscera for advanced carcinoma; a one-stage abdominoperineal operation with end colostomy and bilateral ureteral implantation into the colon above the colostomy. Cancer. $1948 \mathrm{Jul}$; 1(2):177-83.

3. Berek JS1, Howe C, Lagasse LD, Hacker NF. Pelvic exenteration for recurrent gynecologic malignancy: survival and morbidity analysis of the 45-year experience at UCLA. Gynecol Oncol. 2005 0ct;99(1):153-9.

4. Bricker EM. Bladder substitution after pelvic evisceration. Surg Clin North Am. 1950 0ct:30(5):1511-21.

5. Lopez MJ1, Standiford SB, Skibba JL. Total pelvic exenteration. A 50-year experience at the Ellis Fischel Cancer Center. Arch Surg. 1994 Apr;129(4): 390-5; discussion 395-6.

6. Pandey D1, Zaidi S, Mahajan V, Kannan R. Pelvic exenteration: a perspective from a regional cancer center in India. Indian J Cancer. 2004 Jul-Sep; 41(3):109-14.

7. ASTEC study group, Kitchener H, Swart AM, Qian Q, Amos C, Parmar MK. Collaborators (180) Efficacy of systematic pelvic Iymphadenectomy in endometrial cancer (MRC ASTEC trial): a randomised study. Lancet. 2009 Jan 10;373(9658):125-36. doi: 10.1016/S0140-6736(08)61766-3. Epub 2008 Dec 16. Erratum in Lancet. 2009 May 23;373(9677):1764.

8. Benedetti Panici P, Basile S, Maneschi F, Alberto Lissoni A, Signorelli M, Scambia G, et al. Systematic pelvic lymphadenectomy vs. no lymphadenectomy in early-stage endometrial carcinoma: randomized clinical trial. J Natl Cancer Inst. 2008 Dec 3;100(23):1707-16. doi: 10.1093/jnci/ djn397. Epub 2008 Nov 25.

9. Poletto AH, Lopes A, Carvalho AL, Ribeiro EA, Vieira RA, Rossi BM, Aguiar S Jr, Guimaryes GC, Ferreira Fde 0, Nakagawa WT. Pelvic exenteration and sphincter preservation: an analysis of 96 cases. J Surg Oncol. 2004 Jun 1;86(3):122-7.

10. Wydra D, Emerich J, Sawicki S, Ciach K, Marciniak A. Major complications following exenteration in cases of pelvic malignancy: a 10-year experience. World J Gastroenterol. 2006 Feb 21;12(7):1115-9.

11. Randall ME, Filiaci VL, Muss H, Spirtos NM, Mannel RS, Fowler J, et al. Randomized phase III trial of whole-abdominal irradiation versus doxorubicin and cisplatin chemotherapy in advanced endometrial carcinoma: a Gynecologic Oncology Group Study. J Clin Oncol. 2006 Jan 1;24(1):36-44. Epub 2005 Dec 5.

12. Maggioni A, Roviglione G, Landoni F, Zanagnolo V, Peiretti M, Colombo N, et al. Pelvic exenteration: ten-year experience at the European Institute of Oncology in Milan. Gynecol Oncol. 2009 Jul;114(1):64-8. doi: 10.1016/ j.ygyno.2009.03.029. Epub 2009 May 2.

13. Goldberg JM, Piver MS, Hempling RE, Aiduk C, Blumenson L, Recio FO. Improvements in pelvic exenteration: factors responsible for reducing morbidity and mortality. Ann Surg Oncol. 1998 Jul-Aug;5(5):399-406.

14. Nguyen DQ, McGregor AD, Freites 0, Carr ND, Beynon J, El-Sharkawi AM, Lucas MG. Exenterative pelvic surgery--eleven year experience of the Swansea Pelvic Oncology Group. Eur J Surg Oncol. 2005 Dec;31(10): 1180-4. Epub 2005 Aug 26.

15. Barakat RR, Goldman NA, Patel DA, Venkatraman ES, Curtin JP. Pelvic exenteration for recurrent endometrial cancer. Gynecol Oncol. 1999 Oct; 75(1):99-102

16. Lin LL, Mutch DG, Rader JS, Powell MA, Grigsby PW. External radiotherapy versus vaginal brachytherapy for patients with intermediate risk endometrial cancer. Gynecol Oncol. 2007 Jul;106(1):215-20. Epub 2007 May 7.

17. Nout RA, Smit VT, Putter H, Jórgenliemk-Schulz IM, Jobsen JJ, Lutgens LC, et al. Vaginal brachytherapy versus pelvic external beam radiotherapy for patients with endometrial cancer of high-intermediate risk (PORTEC-2): an open-label, non-inferiority, randomised trial. Lancet. 2010 Mar 6;375 (9717):816-23. doi: 10.1016/S0140-6736(09)62163-2. 